\title{
A video-based transdiagnostic REBT universal prevention program for internalizing problems in adolescents: study protocol of a cluster randomized controlled trial
}

Costina Ruxandra Păsărelu ${ }^{1,2}$ and Anca Dobrean ${ }^{1,2^{*}}$

\begin{abstract}
Background: Internalizing problems are the most prevalent mental health problems in adolescents. Transdiagnostic programs are promising manners to treat multiple problems within the same protocol, however, there is limited research regarding the efficacy of such programs delivered as universal prevention programs in school settings. Therefore, the present study aims to investigate the efficacy of a video-based transdiagnostic rational emotive behavioral therapy (REBT) universal prevention program, for internalizing problems. The second objective of the present paper will be to investigate the subsequent mechanisms of change, namely maladaptive cognitions.

Methods: A two-arm parallel randomized controlled trial will be conducted, with two groups: a video-based transdiagnostic REBT universal prevention program and a wait list control. Power analysis indicated that the study will involve 338 participants. Adolescents with ages between 12 and 17 years old, from several middle schools and high schools, will be invited to participate. Assessments will be conducted at four time points: baseline $\left(\mathrm{T}_{1}\right)$, postintervention $\left(T_{2}\right), 3$ months follow-up $\left(T_{3}\right)$ and 12 months follow-up $\left(T_{4}\right)$. Intent-to-treat analysis will be used in order to investigate significant differences between the two groups in both primary and secondary outcomes.

Discussion: This is the first randomized controlled trial that aims to investigate the efficacy and mechanisms of change of a video-based transdiagnostic REBT universal prevention program, delivered in a school context. The present study has important implications for developing efficient prevention programs, interactive, that will aim to target within the same protocol both anxiety and depressive symptoms.
\end{abstract}

Trial registration: ClinicalTrials.gov: NCT02756507. Registered on 25 April 2016.

Keywords: Transdiagnostic, Video-based, Rational emotive behavioral therapy, Internalizing problems, Anxiety, Depression, Universal prevention, Adolescent, School intervention, Mechanisms of change

\footnotetext{
* Correspondence: anca.dobrean@ubbcluj.ro

'Department of Clinical Psychology and Psychotherapy, Babeș-Bolyai

University, Republicii St., No. 37, 400015 Cluj-Napoca, Romania

${ }^{2}$ The International Institute for the Advanced Studies of Psychotherapy and

Applied Mental Health, Republicii St., No. 37, 400015 Cluj-Napoca, Romania
}

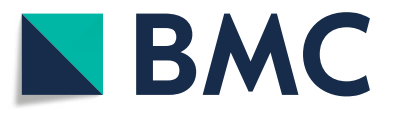

(c) The Author(s). 2018 Open Access This article is distributed under the terms of the Creative Commons Attribution 4.0 International License (http://creativecommons.org/licenses/by/4.0/), which permits unrestricted use, distribution, and reproduction in any medium, provided you give appropriate credit to the original author(s) and the source, provide a link to the Creative Commons license, and indicate if changes were made. The Creative Commons Public Domain Dedication waiver (http://creativecommons.org/publicdomain/zero/1.0/) applies to the data made available in this article, unless otherwise stated. 


\section{Background}

Anxiety and depressive disorders are highly prevalent in adolescents [1]. These disorders, also known as internalizing problems, pose important costs for the adolescent, in terms of affected social relationships, academic achievement, increased suicide risk [2]. The burden of these disorders is high for families and the society [3, 4]. Furthermore, given their chronicity (e.g., anxious disorders) and recurrence (e.g., mood disorders), internalizing problems are persistent $[5,6]$, being often important precursors of these conditions in early adulthood [7]. Internalizing problems in children and adolescents are related to impairments in functionality in the working field in early adulthood [8]. Moreover, along with the negative consequences and the burden of disease, another important aspect that should be considered is that internalizing problems are on the rise. Results of a systematic review showed that as compared with the twentieth century, over the twenty-first century there was an increase in internalizing problems in the adolescent population, especially for girls [9].

Preventing internalizing problems in adolescents could be an important aspect to target, as there is evidence that often anxiety and depressive disorders remain undetected, or even when they are diagnosed, a small percentage of adolescents receive adequate treatment $[10,11]$. Several barriers to access mental health treatments refer to lack of information regarding treatments, stigma, or costs [12]. Another important barrier to receiving mental health treatment is related to adolescents' health-seeking behaviors, namely they fail to recognize their emotional problems, and, consequently, they will not receive adequate treatment [13]. Schools are environments in which adolescents spent a high amount of time. Delivering prevention programs in a school format is cost-effective as shown by a review of Australian school delivered prevention programs for depression [14]. According to a recent metaanalysis, prevention programs delivered in a school context are promising, with small, however significant effects both at short- and long-term follow-up [15].

A main limitation of the existent universal prevention programs is the fact that there are no cross-over effects, specifically only those outcomes that are targeted (e.g., anxiety symptoms in universal prevention programs for anxiety) improve, with no significant changes in the non-targeted condition [16]. These findings call for more research into universal prevention programs that are based on transdiagnostic approaches, targeting both anxiety and depression within the same protocol. Research on transdiagnostic prevention programs delivered in school settings is scarce, with only a few studies being conducted [17-20] that show mixed results regarding the efficacy of transdiagnostic universal prevention programs on anxiety and depression outcomes at posttest, short- and long-term follow-up. However, given the high heterogeneity between these studies (e.g., treatment protocol, design, number of sessions) more research needs to be conducted in order to establish the efficacy of transdiagnostic universal prevention programs, as well as on the investigation of the mechanisms of change involved.

Rational emotive behavior therapy (REBT) [21, 22], a form of cognitive behavioral therapy (CBT), which emphasizes the role of irrational beliefs in generating dysfunctional emotions and maladaptive behaviors, has been previously tested as a prevention program for depressive adolescents [23], or as a brief intervention for adolescents with subclinical internalizing problems [24]. REBT is particularly suitable for a transdiagnostic universal prevention program, given the fact that according to its theory, irrational beliefs (e.g., demandingness, catastrophizing/awfulizing, low frustration tolerance and global evaluation of human worth) are transdiagnostic factors involved in emotional problems [25]. Furthermore, evidence coming from a meta-analysis shows that irrational beliefs are significantly associated with distress [26]. Furthermore, the suitability of REBT for transdiagnostic prevention and interventions programs is also indicated by those studies that show that several specific types of irrational beliefs are involved in multiple disorders (e.g., catastrophizing [27] or frustration intolerance [28]). REBT can be easily adapted for different modalities of delivering interventions, such as technologically enhanced interventions. No study, however, investigated the efficacy of an REBT prevention program delivered via technology enhancements.

Given the existent barriers in the access to mental health services, communication technologies (e.g., videos, text messages, Internet, smartphone apps) can overcome such barriers, improving the availability of mental health services in remote areas and also, reduce the costs [29]. Delivering universal school prevention programs with technological enhancements for adolescents could increase their engagement, improve their adherence to the program, and consequently, influencing the outcomes. For instance, a depression prevention program delivered in an Emergency unit, consisting of PowerPoint presentations supplemented with automated text messages, was found to be acceptable and feasible in a sample of adolescents [30]. Video-based treatments are effective for several conditions and populations, such as insomnia in cancer patients [31], anxiety symptoms in veterans [32], sleep problems in children [33], or aimed to reduce the stigma associated with mental illness in youths [34]. However, as far as we know, there are no studies investigating the efficacy of video-based prevention or intervention programs for adolescents with internalizing problems. Video-based prevention programs 
could be important to investigate, given the fact that videos are more appreciated by participants [35]; another benefit of video-based programs is the fact that they are cost-efficient [36].

\section{Study rationale}

Given recent developments in communication technologies, mental health prevention programs for adolescents could overcome existent barriers in terms of access to evidence-based programs. Universal and indicated prevention programs facilitated through technology (e.g., delivered via smartphones, computers, Internet platforms) are promising in reducing anxiety, depression, and stress in students from higher education [37], with indicated prevention programs delivered with support being associated with better outcomes. Given the fact that most of the universal prevention programs delivered via technology were self-administrated, much research needs to be conducted in order to investigate the efficacy of such programs blended with face-to-face support from a mental health specialist. Moreover, the high comorbidity between anxiety and depressive disorders calls for the development and investigation of more efficient programs, which can target multiple problems within the same protocol. There is evidence that transdiagnostic interventions facilitated via technology are associated with moderate to large effect sizes in adult populations in what regards anxiety, depression, and quality of life outcomes [38]. Nonetheless, when considering young populations, research is scarce, with mixed results, few randomized controlled trials conducted and many confounding variables existing regarding the efficacy of transdiagnostic universal prevention programs facilitated via technology for anxiety and depression in adolescents. Schools are ecological environments that can be used in order to reach to a high number of adolescents, however much research needs to be conducted in order to investigate the efficacy of school-delivered universal prevention programs. There is a need for conducting more randomized controlled trials with larger samples of adolescents that could inform research not only about the effect that such a prevention program might have on adolescent related outcomes (e.g., reductions in anxiety, depression symptoms), but also, on the mechanisms of change, namely the active ingredients through which the intervention can influence those outcomes. Video-based programs have been so far tested as self-administrated interventions in both adult [31,32] and young populations [33, 34], for health and emotional problems. Moreover, they hold great promises in terms of access to evidence-based programs, interactive content that can increase participants' engagement and adherence to the program, limited input from the person that delivers the intervention.

\section{Aim}

The present study aims to investigate the efficacy of a video-based transdiagnostic REBT universal prevention program for internalizing problems in Romanian adolescents, implemented in a school setting. Our second aim is to investigate subsequent mechanisms of change, namely, we want to investigate whether maladaptive cognitions are significant mediators of the prevention program's efficacy.

\section{Methods/Design}

The study design is reported according to the CONSORT 2010 (Consolidated Standards of Reporting Trials) Statement [39] and the SPIRIT 2013 (Standard Protocol Items: Recommendations for Interventional Trials) Statement [40].

\section{Design}

A two-arm cluster randomized controlled trial (RCT) will be conducted. Participants' school classes will be randomized by a computer software (www.randomizer.org) in one of the two treatment conditions: (1) video-based transdiagnostic REBT universal prevention program or (2) waitlist control. Assessments will be conducted at four time points: baseline $\left(T_{1}\right)$, post-intervention $\left(T_{2}\right), 3$ months follow-up $\left(\mathrm{T}_{3}\right)$ and 12 months follow-up $\left(\mathrm{T}_{4}\right)$. Figure 1 illustrates the flowchart of the study.

\section{Participants \\ Recruitment}

Adolescents will be recruited from several public schools, namely from middle schools and high schools from Central, East and Nord West cities from Romania. School principals will be invited to participate and invitation letters containing information about the study will be offered to parents and adolescents.

\section{Eligibility criteria \\ Inclusion criteria}

In order to be eligible for the present study, several inclusion criteria have been established:

(1) Preadolescents and adolescents will have ages between 12 and 17 years old;

(2) Are attending middle school or high-school;

(3) Adolescents have sufficient understanding of the Romanian language in order to complete assessments at all time points and to participate in the prevention program;

(4) Their parents have sufficient understanding of the Romanian language in order to sign the informed consent;

(5) Parents will sign the informed consent and adolescents will provide informed assent. 


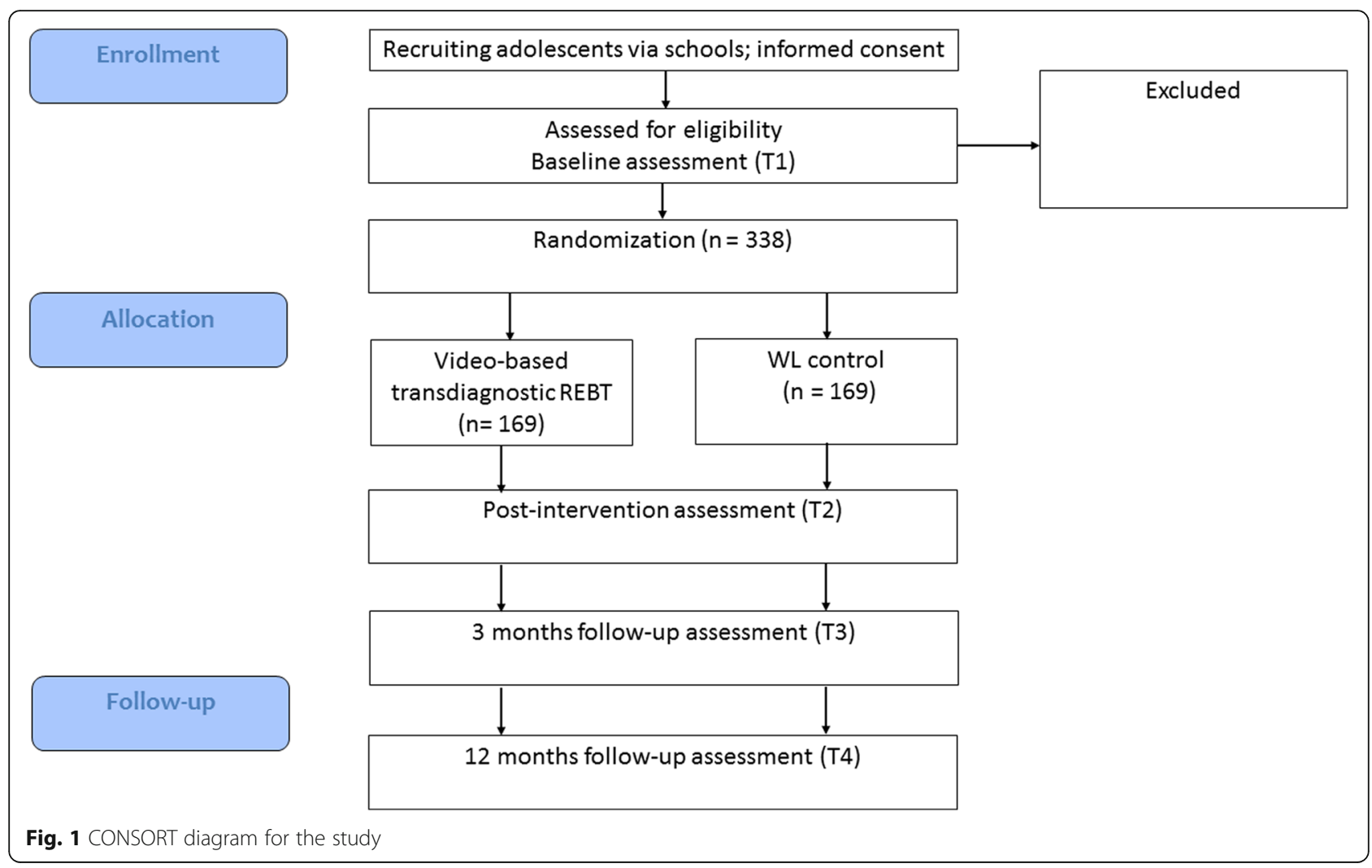

\section{Exclusion criteria}

(1) Age below 12 or above 17 years;

(2) No signed informed consent from parents;

(3) Adolescents will score high on suicidal ideation according to (1 item on the Depression subscale from Beck Youth Inventories [41]);

(4) Not Romanian-speaking in order to understand the content of the prevention program and to complete the assessments.

\section{Randomization and blinding}

Randomization will be applied within classes, using a random numbers generator (www.randomizer.org), with an equal number of experimental and control groups within one school. Children will be assigned to either videobased transdiagnostic REBT universal prevention or to waitlist control condition. Allocation concealment will be done by an independent researcher, using opaque, sealed envelops that will be opened sequentially. Blinding of participants, researchers and assessors will be maintained.

\section{Sample size calculation}

A priori power analyses using $G^{*}$ Power version 3.1.5 [42] indicated that a total sample size of 260 would be sufficient to detect a small to medium effect size $(d=.35)$ according to the results or previous research, for a two-tails between-group comparison at posttest, with an alpha of .05 and a power of $80 \%$. As we expected to have a $30 \%$ dropout rate, we will recruit 169 participants in each group.

\section{Study conditions}

\section{The wait-list control group}

Participants in the waiting list will not receive an intervention. They will complete assessments at all four time points. They could receive the intervention after the experimental group has completed all the assessments.

\section{Video-based transdiagnostic REBT prevention program}

The authors, both being certified in REBT from Albert Ellis Institute, New-York, have developed a transdiagnostic REBT protocol for this study by adjusting existent protocols for internalizing problems in adolescents [43, 44]. Namely, our protocol was based on REBT theory [21]. Further, we shortened existent programs in order to investigate the efficacy of a briefer version of the protocol. The prevention program will be delivered in the school context, namely in adolescent's school classes after regular school hours. The program will consist of six $50 \mathrm{~min}$ sessions of video-based REBT (See Table 1 for a content of each session) delivered for three weeks (two times per week) in a group format. Research assistants (Masters students, psychologists trained in CBT) will present each 
Table 1 Protocol contents for each session

\begin{tabular}{ll}
\hline Session number & Content \\
\hline 1 & Psychoeducation regarding internalizing problems \\
3 & How to identify anxiety and depression. Relaxation \\
4 & The ABC model of distress \\
5 & Cognitive restructuring \\
6 & Exposure and behavioral activation. Problem solving \\
& Maintenance of therapeutic gains and relapse \\
\hline
\end{tabular}

session based on a video cartoon and after this, a standardized PowerPoint presentation will be used to guide the discussion with the adolescents. They will learn various aspects related to internalizing problems, how irrational beliefs influence dysfunctional emotions, how to construct a hierarchy of feared situations and how to expose gradually. Also, they will learn breathing and relaxation exercises. Between sessions, homework will be provided and will be checked at the beginning of each session. The content of each session is presented as follows:

Session 1. Psychoeducation regarding internalizing problems. In this session, adolescents will be provided with information regarding internalizing problems. Videos regarding different internalizing symptoms will be presented and discussions regarding different types of symptoms will be initiated with the adolescents. Also, they will be informed about the program and about their involvement in this program.

Session 2. Anxious and depressive symptoms identification and relaxation exercises. In this session, adolescents will learn how to recognize anxiety and depression. Also, they will learn the four levels on which such emotions appear: physiological, subjective, cognitive, and behavioral. They will learn the importance of relaxation exercises in order to decrease physiological arousal and they will practice in class a relaxation exercise.

Session 3. The $A B C$ model of distress. In this session, adolescents will be thought the $A B C$ model of distress, where A stands for activating events, B stands for beliefs and $\mathrm{C}$ stands for consequences. They will learn to distinguish between rational and irrational beliefs, functional and dysfunctional emotions, and discover how different irrational beliefs (e.g., demandingness, awfulizing/catastrophizing, low frustration tolerance, global evaluation of human worth) are related to different negative dysfunctional emotions (e.g., anxiety, depression) according to the REBT theory. Finally, they will practice examples in which different activating situations are related to different emotions through the influence of irrational beliefs.

Session 4. Cognitive restructuring. In this session, adolescents will identify, learn how to dispute their irrational beliefs logically (e.g., "Is your belief logical?"), empirically (e.g., "Is there any evidence sustaining your belief?") and pragmatically (e.g., "Is this belief helpful in any way for you?"). Finally, participants will learn how to dispute their irrational beliefs (demandingness, catastrophizing/ awfulizing, low frustration tolerance and global evaluation of human worth) and to replace them with rational beliefs (preference beliefs, non-catastrophizing, high frustration tolerance, unconditional acceptance) associated with functional emotions and adaptive behaviors. Namely, in this session, the $A B C$ model is integrated with other two important components, namely Disputation (D) and Effective new responses (E) (e.g., functional emotions and adaptive behaviors associated with the new beliefs), thus turning into the extended ABCDE model.

Session 5. Exposure, behavioral activation and problemsolving. In this session, adolescents will learn how avoidance perpetuates anxiety, and the difference between exposure and avoidance. They will build an exposure hierarchy comprised of fear ladders, with a gradual increase of the distress after disputing their irrational beliefs (e.g., irrational beliefs about the confrontation with feared stimuli). The will learn the role of behavioral activation in decreasing depressive mood and will be encouraged to do enjoyable things in order to decrease their depressive symptoms. The second part of this session, adolescents will learn the difference between emotional and practical problems. In this sense, they will learn how to solve practical problems by applying a problem-solving protocol.

Session 6. Maintenance of therapeutic gains and relapse prevention. Adolescents will learn how to set realistic expectations regarding the occurrence of internalizing symptoms, and instead of thinking that they will never feel anxiety or depression they will learn that negative emotions can occur, however by identifying and restructuring their irrational beliefs, they will be able to turn those negative dysfunctional emotions into negative functional emotions (e.g., worry, sadness). Finally, the last session will be a booster session, in which a summary of the program will be presented in order to review all the strategies that adolescents acquired throughout the prevention program so as to prepare them to handle future challenges.

Homework assignments will be given after each session and participants will have to monitor their emotions (e.g., functional and dysfunctional emotions), their thinking patterns (e.g., identify the $\mathrm{ABC}$ components from personal examples), dispute their irrational beliefs and replace them with rational beliefs (e.g., the expanded ABCDE model), practice relaxation exercises (e.g., deep breathing, progressive muscle relaxation) at home and in other settings, practice exposure in imaginary and in vivo (e.g., behavioral assessments such as shame-attacking exercises), practice behavioral activation. 


\section{Therapists}

Therapists $(N=5)$ will be Masters Students, psychologists trained in CBT, under supervision. Therapists will be trained before conducting the sessions with the adolescents and weekly supervision sessions will be conducted with the team.

\section{Data collection}

Adolescents will complete all the assessments at school, in their classrooms, in the presence of a research assistant, blind to group allocation, that will provide assistance if needed. All the instruments will be applied in a paper and pencil format. Adolescents will complete assessments at four time-points: $\mathrm{T}_{1}$ (baseline), $\mathrm{T}_{2}$ (post-intervention), $\mathrm{T}_{3} \quad$ (3 months follow-up) and $\mathrm{T}_{4}$ (12 months follow-up). An overview of the instruments that will be used for baseline, post-treatment, 3 months follow-up and 12 months follow-up is provided in Fig. 2.

\section{Outcomes}

\section{Demographic data}

Both adolescents and their parents will complete a questionnaire regarding their demographic characteristics, such as gender, age, urban or rural residency and completed years of education.

\section{Primary outcomes}

\section{Anxiety symptoms}

The Multidimensional Anxiety Scale for Children (MASC) [45] will be used to assess anxiety symptoms in adolescents. This instrument consists of 39 items, rated on a four-point Likert scale, where 0 means Never true and 3 means Often true. This scale has four subscales, namely: Physical Symptoms, Social Anxiety, Harm Avoidance and Separation Anxiety/Panic.

\section{Depressive symptoms}

The depression subscale of the Beck Youth Inventories $^{\mathrm{Tm}}$-Second Edition for Children and Adolescents

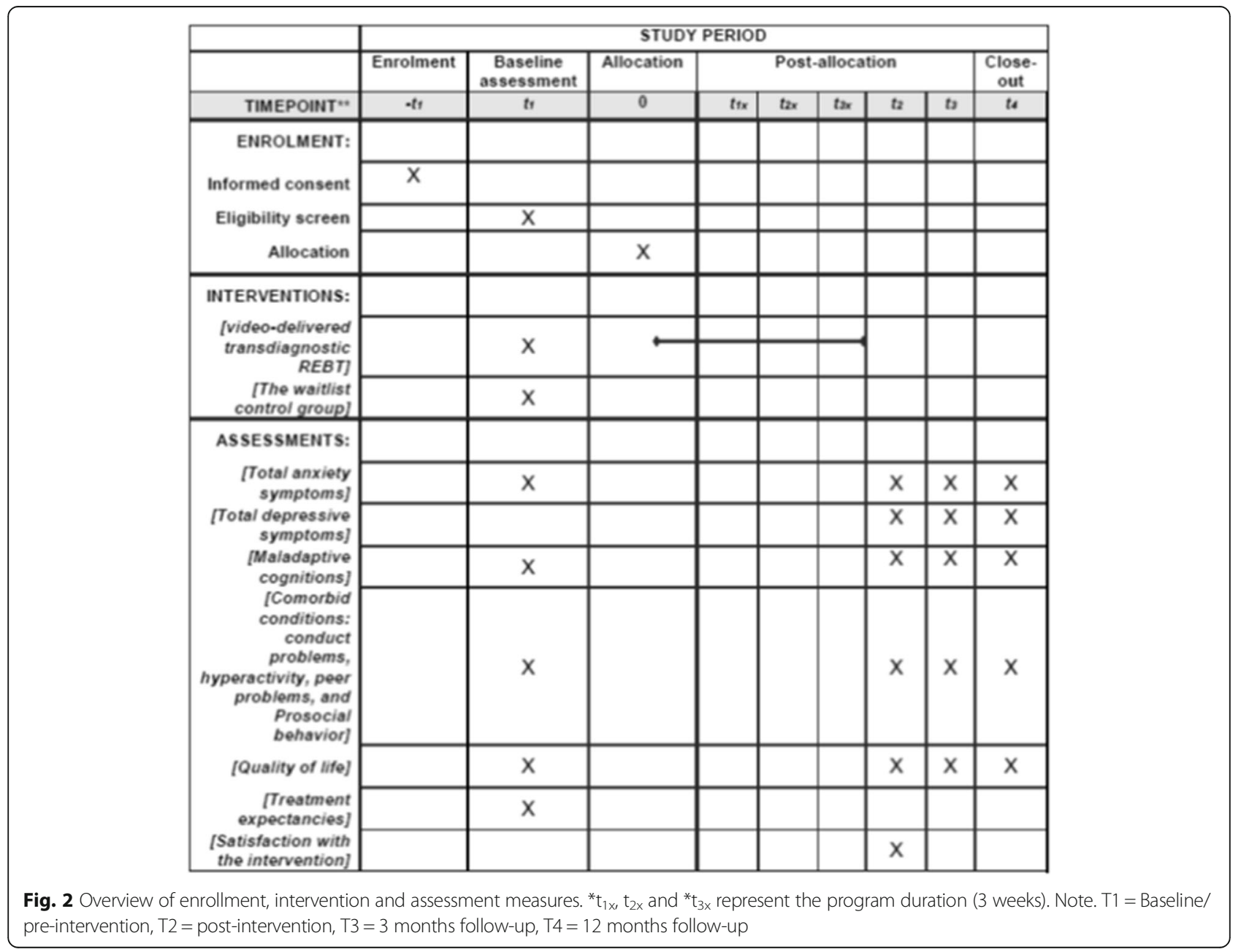


(BYI-II) [41] will be used to assess adolescents' depressive symptoms. BYI-II can be used with children and adolescents with ages between 7 and 18 years. This subscale contains 20 items, rated on a 4 point Likert scale, with $0=$ Never and $3=$ Always. The total score is obtained by summing the 20 items.

\section{Secondary outcomes Comorbid problems}

The Strengths and Difficulties Questionnaire (SDQ) [46] self-reported version for youths aged 11-17 will be used in this study. SDQ consists of 25-items, rated on a three-point Likert scale, where 0 means Not true and 2 means Certainly true. This instrument has five scales: Emotional problems, Conduct problems, Hyperactivity, Peer problems, and Prosocial behavior. This scale allows to compute a Total difficulties score, by summing the first three previously mentioned scales; also, two total scores for Internalizing (by adding emotional and peer problems scales) and Externalizing (by adding conduct and hyperactivity scales) can be computed [47]. We will use the Externalizing problems scale in order to investigate whether the program will have a significant impact on comorbid problems that adolescents may present. Also, we will use the Prosocial behavior subscale in order to investigate potential significant changes in positive outcomes.

\section{Maladaptive cognitions}

The Children's Automatic Thoughts Scale - Negative/ Positive (CATS-N/P) [48] will be used to assess negative and positive automatic thoughts in adolescents. CATS$\mathrm{N} / \mathrm{P}$ consists of 50 items, rated on a 5 point Likert scale, where 0 means Not at all and 4 means All the time. Total negative automatic thoughts score is computed by summing the three subscales, namely: Physical Threat, Social Threat, and Personal Failure, consisting of 40 items, while total positive automatic thoughts scale consists of 10 items. This instrument showed adequate psychometric properties (e.g., internal consistency, discriminant validity) previously [48].

\section{Quality of life}

The Questionnaire for Measuring Health-Related Quality of Life in Children and Adolescents, The Revised Version - adolescent version (Kiddo-KINDL) [49] will be used to assess adolescents' quality of life. KINDL-R-a consists of 24 items that can be used with children and adolescents aged 3-17 years old. It has six subscales with four items each (physical and emotional well-being, self-esteem, family, friends, and school), all items scored on a 5 -point scale.

\section{Treatment expectancies}

We translated and adapted The Credibility/ Expectancy Questionnaire [50] in order to use this instrument to assess adolescent's expectancies regarding the prevention program. This instrument consists of six items, four of them scored on a 9 point Likert scale $(1=$ Not at all, $9=$ Very much) while for two items participants will have to choose a percentage between $0 \%$ and $100 \%$ for expected improvement in their problems.

\section{Satisfaction with the intervention}

The Romanian version of the Client Satisfaction Scale, that has been previously used to assess satisfaction with an Internet-delivered CBT [51], will be used in order to assess participants' satisfaction with the intervention. This scale consists of 10 items rated on a 5 point Likert scale $(1=$ Don't agree at all and $5=$ Very much agree $)$. Higher scores on the Satisfaction with treatment scale will indicate greater levels of satisfaction with the program.

\section{Statistical methods}

Statistical analyses were conducted using SPSS 23.0 software. First, independent t-tests and Chi-Square tests will be conducted in order to explore potential differences between participants from the two groups regarding age, gender, anxiety, depressive symptoms, irrationality, comorbid conditions, and quality of life. At post-treatment (T2), three months follow-up (T3) and twelve months follow-up (T4), continuous analysis will be conducted using intent-to-treat (ITT) principle the last observation carried forward. In order to assess the efficacy of the video-based transdiagnostic REBT universal prevention program to reduce anxiety and depressive outcomes as compared with the control group over time, we will use mixed effects models (random intercept and slope for time, a fixed effect for group). For effect size data we will use Cohen's $d$ (small effect size $d=.20$, medium effect size $d=.50$, large effect size $d=.80 ;$ [52]). Mediation and moderation analyses will be conducted using PROCESS macro [53].

\section{Monitoring study implementation}

All the group sessions will be conducted in schools by five registered psychologists, Masters Students. Weekly supervision sessions will be conducted with the therapists in order to solve potential issues that could result in the administration of the intervention. Treatment fidelity and therapists' adherence to the protocol will be assessed by randomly listening to audio recordings of the sessions. 


\section{Ethics and dissemination}

This trial will be conducted in compliance with the Declaration of Helsinki [54]. The study was approved by the Ethics Committee of Babeș-Bolyai University (Registration number: 33215/ 03.06.2016). In Table 2 is presented the trial registration dataset as recommended by World Health Organisation (WHO). Prior to assessment, parents will sign the informed consent, agreeing for their offsprings to

Table 2 WHO Trial Registration Data Set

\begin{tabular}{|c|c|}
\hline Data category & Information \\
\hline Primary registry and trial identifying number & ClinicalTrials.gov NCT02756507 \\
\hline Date of registration in primary registry & 25 April 2015 \\
\hline Secondary identifying numbers & The Ethics Committee of Babeș-Bolyai University (Registration number: 33215/ 03.06.2016) \\
\hline Sources of monetary or material support & $\begin{array}{l}\text { The Romanian Executive Unit for Financing Education Higher Research, Development and } \\
\text { Innovation, Babeș-Bolyai University }\end{array}$ \\
\hline Primary sponsor & Babeș-Bolyai University \\
\hline Contact for public queries & $\begin{array}{l}\text { Costina Ruxandra Păsărelu } \\
\text { Department of Clinical Psychology and Psychotherapy } \\
\text { Babeș-Bolyai University } \\
\text { Romania } \\
\text { Republicii St., No. } 37 \\
\text { Postal code: } 400015 \\
\text { E-mail: costina.pasarelu@ubbcluj.ro }\end{array}$ \\
\hline Contact for scientific queries & $\begin{array}{l}\text { Prof. Anca Dobrean } \\
\text { Babeș-Bolyai University } \\
\text { Republicii St., No. } 37 \\
\text { Postal code: } 400015 \\
\text { E-mail: anca.dobrean@ubbcluj.ro }\end{array}$ \\
\hline Public title & Transdiagnostic REBT Prevention Program for Adolescents \\
\hline Scientific title & $\begin{array}{l}\text { A video-based transdiagnostic REBT universal prevention program for internalizing problems } \\
\text { in adolescents }\end{array}$ \\
\hline Countries of recruitment & Romania \\
\hline Health condition(s) or problem(s) studied & $\begin{array}{l}\text { Anxiety } \\
\text { Depression } \\
\text { Internalizing problems }\end{array}$ \\
\hline Intervention(s) & $\begin{array}{l}\text { Experimental: video-based transdiagnostic REBT prevention program } \\
\text { Control: waiting list }\end{array}$ \\
\hline Key inclusion and exclusion criteria & $\begin{array}{l}\text { Inclusion criteria: Preadolescents and adolescents will have ages between } 12 \text { and } 17 \text { years old; } \\
\text { Are attending middle school or high-school; Adolescents have sufficient understanding of the } \\
\text { Romanian language in order to complete assessments at all time points and to participate in } \\
\text { the prevention program; Their parents have sufficient understanding of the Romanian language } \\
\text { in order to sign the informed consent; Parents will sign the informed consent and adolescents } \\
\text { will provide informed assent. } \\
\text { Exclusion criteria: Age below } 12 \text { or above } 17 \text { years; No signed informed consent from parents; } \\
\text { Adolescents will score high on suicidal ideation according to ( } 1 \text { item on the Depression subscale } \\
\text { from Beck Youth Inventories); Not Romanian-speaking in order to understand the content of the } \\
\text { prevention program and to complete the assessments. }\end{array}$ \\
\hline Study type & Randomized controlled trial \\
\hline Date of first enrollment & June 2016 \\
\hline Target sample size & 338 \\
\hline Recruitment status & Recruiting \\
\hline Primary outcome(s) & $\begin{array}{l}\text { Anxiety symptoms at } T_{2}, T_{3}, T_{4} \text {; the Multidimensional Anxiety Scale for Children } \\
\text { Depressive symptoms at } T_{2}, T_{3}, T_{4} \text {; the Beck Youth Inventories }{ }^{\mathrm{TM}} \text {-Second Edition for Children and } \\
\text { Adolescents }\end{array}$ \\
\hline Key secondary outcome(s) & $\begin{array}{l}\text { Maladaptive cognitions at } T_{2}, T_{3}, T_{4} \text {; the Children's Automatic Thoughts Scale - Negative/Positive } \\
\text { Comorbid problems at } T_{2}, T_{3}, T_{4} \text {; the Strengths and Difficulties Questionnaire } \\
\text { Prosocial behaviors } \\
\text { Quality of life at } T_{2}, T_{3}, T_{4} \text {; the Questionnaire for Measuring Health-Related Quality of Life in } \\
\text { Children and Adolescents, The Revised Version - adolescent version } \\
\text { Treatment expectancies at } T_{2} \text {; the Credibility/ Expectancy Questionnaire } \\
\text { Satisfaction with the intervention at } T_{2} ; \text { the Client Satisfaction Scale }\end{array}$ \\
\hline
\end{tabular}


participate in the trial. Adolescents will sign the informed assent to participate in the study. Assessment reports contain no personal identifying information. No incentives will be offered for participation. Adolescents will be informed that they can withdraw from the study at any moment.

\section{Dissemination policy}

This trial's results will be submitted for publication in peer-reviewed journals, focusing on primary and secondary outcomes results, as well as on mechanisms of change. Also, results will be presented at national and international conferences.

\section{Discussion}

Internalizing problems, namely anxiety and depressive disorders are prevalent conditions in adolescents [1], associated with a high burden of disease for families, as well as for the society [3, 4]. Despite the high prevalence and the associated costs of internalizing problems, the problem of treatment accessibility is an important one. In fact, most of these disorders remain undetected and, consequently, untreated, but what is even more, is the fact that even when adolescents receive mental health treatment, only a few receive adequate services $[10,11]$. Barriers to the access to adequate mental health care could be tacked if prevention programs would be delivered in school contexts. Universal school delivered prevention programs are great modalities to prevent the onset of youths' anxiety and depression disorders, two of the most common problems that emerge in adolescence. Therefore, by targeting both anxiety and depressive disorders in a school context, adolescents could learn about their symptoms and benefit on such programs. Also, another considerable barrier to accessing mental health services, namely stigma, would be reduced, as all the adolescents will receive the same program, and their mental health literacy will be improved. Furthermore, youths' predilection for using technology should be taken into consideration when designing such programs, in order to improve their adherence. Existent advances in information and communication technologies can help to overcome the barriers to the access to mental health services. The aim of the present study is to investigate the efficacy of a video-based transdiagnostic REBT universal prevention program delivered in a school setting as compared with a waiting list control. Furthermore, a second aim of the present study is to investigate adolescents' irrational beliefs as mediators, namely subsequent mechanisms of change of the prevention program.

There are several limitations that we should consider. First, as we rely only on self-reported data, potential bias can result in the case of adolescents. Another important limitation could be the lack of a placebo condition.
However, as this will be the first trial comparing the efficacy of a transdiagnostic video-delivered REBT prevention program, we aim to compare it with a waitlist condition and intend to extend this study and compare it with an active placebo or a traditional face-to-face program. Another limitation is related to the possible dropout at 12-month follow-up assessment, as it is possible that several adolescents entering high school will change their schools or live in a different city.

In conclusion, this is the first study that aims to investigate the efficacy of a video-based transdiagnostic REBT universal prevention program in reducing internalizing problems in adolescents. Also, it is the first study that integrated REBT with technology in the format of a video-based program aimed to reduce anxiety and depression outcomes in youths. Video-based interventions take into consideration young peoples' predilection to use technology.

Moreover, as indicated in a recent review study analyzing school mental health programs delivered for more than 27 million of children [55], most of the research on the efficacy of prevention programs for children delivered in school contexts was conducted in high-income countries. We, therefore, need to conduct such a study in a Romanian population of adolescents, given the limited existent research on the efficacy of such programs in emerging countries.

\section{Trial status}

Participants' recruitment is still ongoing.

\section{Abbreviations}

ANOVA: Analyses of variance; BYI-II: Beck Youth Inventories ${ }^{\mathrm{TM}}$-Second Edition for Children and Adolescents; CATS-N/P: The Children's Automatic Thoughts Scale - Negative/Positive; CBT: Cognitive behavioral therapy; ITT: Intent-totreat; KINDL-R-a: The Questionnaire for Measuring Health-Related Quality of Life in Children and Adolescents, The Revised Version; MASC: The Multidimensional Anxiety Scale for Children; REBT: Rational emotive behavioral therapy; RM: Repetead measures (RM); SDQ: The Strengths and Difficulties Questionnaire; WL: Wait list

\section{Acknowledgments}

Not applicable

\section{Funding}

This work was supported by a grant from the Romanian Executive Unit for Financing Education Higher Research, Development and Innovation (the "Effectiveness of an empirically based web platform for anxiety in youths", [grant number PN-II-PT-PCCA-2011-3.1-1500, 81/2012]) awarded to Dr. Anca Dobrean.

\section{Availability of data and materials}

Upon the completion of the study, the datasets will be available from the corresponding author on reasonable request.

\section{Authors' contributions}

All authors participated in the design of the study. AD obtained funding. CPR drafted the manuscript and AD revised the manuscript. Both authors read and approved the final manuscript. 


\section{Authors' information}

CPR is an Assistant Professor at the Department of Clinical Psychology and Psychotherapy of Babeș-Bolyai University, Cluj-Napoca, Romania. Her main interests regard transdiagnostic treatments delivered via technology for youth populations as well as for parents. AD is a Professor at the Department of Clinical Psychology and Psychotherapy of Babeș-Bolyai University, ClujNapoca, Romania. She is the director of the Infant, Child, and Adolescent Research Program. Her main interest regard evidence-based assessment and evidence-based interventions for children and adolescents.

\section{Ethics approval and consent to participate}

Written informed consent will be obtained from parents of all adolescents involved in the study prior to their involvement. The study was approved by the Ethics Committee of Babeș-Bolyai University (Registration number: 33215/ 03.06.2016). The study was also prospectively registered at ClinicalTrials.gov (NCT02756507, 25 April 2015).

\section{Competing interests}

The authors declare that they have no competing interests.

\section{Publisher's Note}

Springer Nature remains neutral with regard to jurisdictional claims in published maps and institutional affiliations.

Received: 24 July 2017 Accepted: 3 April 2018 Published online: 13 April 2018

\section{References}

1. Polanczyk GV, Salum GA, Sugaya LS, Caye A, Rohde LA. Annual research review: a meta-analysis of the worldwide prevalence of mental disorders in children and adolescents. J Child Psychol Psychiatry. 2015;56:345-65.

2. Balázs J, Miklósi M, Keresztény Á, Hoven CW, Carli V, Wasserman C, et al. Adolescent subthreshold-depression and anxiety: psychopathology, functional impairment and increased suicide risk. J Child Psychol Psychiatry. 2013;54:670-7

3. Gallo KP, Olin SS, Storfer-Isser A, O'Connor BC, Whitmyre ED, Hoagwood KE, et al. Parent burden in accessing outpatient psychiatric Services for Adolescent Depression in a large state system. Psychiatr Serv. 2016;68:411-4.

4. Pape K, Bjørngaard JH, Holmen TL, Krokstad S. The welfare burden of adolescent anxiety and depression: a prospective study of 7500 young Norwegians and their families: the HUNT study. BMJ Open. 2012;2:e001942.

5. Ormel J, Raven D, van Oort F, Hartman CA, Reijneveld SA, Veenstra R, et al. Mental health in Dutch adolescents: a TRAILS report on prevalence, severity, age of onset, continuity and co-morbidity of DSM disorders. Psychol Med. 2015;45:345-60.

6. Snyder HR, Young JF, Hankin BL. Strong homotypic continuity in common psychopathology-, internalizing-, and externalizing-specific factors over time in adolescents. Clin Psychol Sci J Assoc Psychol Sci. 2017;5:98-110.

7. Patton GC, Coffey C, Romaniuk H, Mackinnon A, Carlin JB, Degenhardt L, et al. The prognosis of common mental disorders in adolescents: a 14-year prospective cohort study. Lancet. 2014;383:1404-11.

8. Narusyte J, Ropponen A, Alexanderson K, Svedberg P. Internalizing and externalizing problems in childhood and adolescence as predictors of work incapacity in young adulthood. Soc Psychiatry Psychiatr Epidemiol. 2017;

9. Bor W, Dean AJ, Najman J, Hayatbakhsh R. Are child and adolescent mental health problems increasing in the 21st century? A systematic review. Aust N Z J Psychiatry. 2014;48:606-16.

10. Chavira DA, Stein MB, Bailey K, Stein MT. Child anxiety in primary care: prevalent but untreated. Depress Anxiety. 2004;20:155-64.

11. Stein K, Fazel M. Depression in young people often goes undetected. The Practitioner. 2015;259:17-22. 2-3

12. Salloum A, Johnco C, Lewin AB, McBride NM, Storch EA. Barriers to access and participation in community mental health treatment for anxious children. J Affect Disord. 2016;196:54-61.

13. Gulliver A, Griffiths KM, Christensen H. Perceived barriers and facilitators to mental health help-seeking in young people: a systematic review. BMC Psychiatry. 2010;10:113

14. Lee YY, Barendregt JJ, Stockings EA, Ferrari AJ, Whiteford HA, Patton GA, et al. The population cost-effectiveness of delivering universal and indicated school-based interventions to prevent the onset of major depression among youth in Australia. Epidemiol Psychiatr Sci. 2016:1-20.
15. Werner-Seidler A, Perry Y, Calear AL, Newby JM, Christensen H. Schoolbased depression and anxiety prevention programs for young people: a systematic review and meta-analysis. Clin Psychol Rev. 2017:51:30-47.

16. Garber J, Brunwasser SM, Zerr AA, Schwartz KTG, Sova K, Weersing VR. Treatment and prevention of depression and anxiety in youth: test of crossover effects. Depress. Anxiety. 2016;33:939-59.

17. Greca AML, Ehrenreich-May J, Mufson L, Chan S. Preventing adolescent social anxiety and depression and reducing peer victimization: intervention development and open trial. Child Youth Care Forum. 2016;45:905-26.

18. Johnson C, Burke C, Brinkman S, Wade T. Effectiveness of a school-based mindfulness program for transdiagnostic prevention in young adolescents. Behav Res Ther. 2016;81:1-11.

19. Martinsen KD, Kendall PC, Stark K, Neumer S-P. Prevention of anxiety and depression in children: acceptability and feasibility of the Transdiagnostic EMOTION program. Cogn Behav Pract. 2016:23:1-13.

20. Nehmy TJ, Wade TD. Reducing the onset of negative affect in adolescents: evaluation of a perfectionism program in a universal prevention setting. Behav Res Ther. 2015;67:55-63.

21. Ellis A. Reason and emotion in psychotherapy. Oxford, England: Lyle Stuart, 1962.

22. Ellis A. Reason and emotion in psychotherapy, revised. Secaucus, Birch Lane, 1994.

23. Marcotte $D$, Baron P. L'efficacité d'une stratégie d'intervention émotivorationnelle auprès d'adolescents dépressifs du millieu scolaire. Can. J. Couns. Psychother. Rev. Can. Couns. Psychothérapie. 1993 [cited 2017 Jul 16];27. Available from: http://cjcp.journalhosting.ucalgary.ca/cjc/index.php/rcc/ article/view/545

24. Sælid GA, Nordahl HM. Rational emotive behaviour therapy in high schools to educate in mental health and empower youth health. A randomized controlled study of a brief intervention. Cogn Behav Ther. 2017:46:196-210.

25. Taylor S, Clark DA. Transdiagnostic cognitive-behavioral treatments for mood and anxiety disorders: introduction to the special issue. J Cogn Psychother. 2009;23:3-5

26. Vî̀lă A, Flückiger C, Grosse HM, David D. Irrational beliefs and psychological distress: a meta-analysis. Psychother Psychosom. 2016;85:8-15.

27. Gellatly R, Beck AT. Catastrophic thinking: a Transdiagnostic Process across psychiatric disorders. Cogn Ther Res. 2016;40:441-52.

28. Harrington N. Frustration intolerance beliefs: their relationship with depression, anxiety, and anger, in a clinical population. Cogn Ther Res. 2006:30:699-709.

29. Jones E, Manassis K, Arnold P, Ickowicz A, Mendlowitz S, Nowrouzi B, et al. Translating cognitive behavioral therapy for anxious youth to rural-community settings via tele-psychiatry. Community Ment Health J. 2015;51:852-6.

30. Ranney ML, Freeman JR, Connell G, Spirito A, Boyer E, Walton M, et al. A depression prevention intervention for adolescents in the emergency department. J Adolesc Health. 2016;59:401-10.

31. Savard J, Ivers H, Savard M-H, Morin CM. Is a video-based cognitive behavioral therapy for insomnia as efficacious as a professionally administered treatment in breast cancer? Results of a randomized controlled trial. Sleep. 2014;37:1305-14.

32. Gould CE, Zapata AML, Bruce J, Merrell SB, Wetherell JL. O\&\#39 R, et al. development of a video-delivered relaxation treatment of late-life anxiety for veterans. Int Psychogeriatr. 2017:1-13.

33. Surani SR, Surani SS, Sadasiva S, Surani Z, Khimani A, Surani SS. Effect of animated movie in combating child sleep health problems. SpringerPlus. 2015:4:343.

34. Janoušková M, Tušková E, Weissová A, Trančík P, Pasz J, Evans-Lacko S, et al. Can video interventions be used to effectively destigmatize mental illness among young people? A systematic review. Eur Psychiatry J Assoc Eur Psychiatr. 2017:41:1-9.

35. Walthouwer MJL, Oenema A, Lechner L, de Vries H. Comparing a Video and Text Version of a Web-Based Computer-Tailored Intervention for Obesity Prevention: A Randomized Controlled Trial. J. Med. Internet Res. 2015;17. Available from: http://www.ncbi.nlm.nih.gov/pmc/articles/ PMC4642381/

36. Stanczyk NE, Smit ES, Schulz DN, de Vries H, Bolman C, Muris JWM, et al. An economic evaluation of a video- and text-based computer-tailored intervention for smoking cessation: a cost-effectiveness and cost-utility analysis of a randomized controlled trial. PLoS One. 2014;9:e110117.

37. Conley CS, Durlak JA, Shapiro JB, Kirsch AC, Zahniser E. A Meta-Analysis of the Impact of Universal and Indicated Preventive Technology-Delivered 
Interventions for Higher Education Students. Prev. Sci. Off. J. Soc. Prev Res. 2016;17:659-678.

38. Păsărelu CR, Andersson G, Bergman Nordgren L, Dobrean A. Internetdelivered transdiagnostic and tailored cognitive behavioral therapy for anxiety and depression: a systematic review and meta-analysis of randomized controlled trials. Cogn Behav Ther. 2017:46:1-28.

39. Schulz KF, Altman DG, Moher D. CONSORT 2010 statement: updated guidelines for reporting parallel group randomised trials. Trials. 2010;1 1:32

40. Chan A-W, Tetzlaff JM, Altman DG, Laupacis A, Gøtzsche PC, Krleža-Jerić K, et al. SPIRIT 2013 statement: defining standard protocol items for clinical trials. Ann Intern Med. 2013;158:200-7.

41. Beck Youth Inventories ${ }^{\mathrm{TM}}$ - Second Edition [Internet]. [cited 2017 Jul 16]. Available from: http://www.pearsonclinical.com/psychology/products/ 100000153/beck-youth-inventories-second-edition-byi-ii.html

42. Faul F, Erdfelder E, Lang A-G, Buchner A. G*power 3: a flexible statistical power analysis program for the social, behavioral, and biomedical sciences. Behav Res Methods. 2007:39:175-91.

43. Cristea IA, Stefan S, David O, Mogoase C, Dobrean A. Rational-Emotive and Cognitive-Behavior Therapy (RE\&amp;BT) Treatment Protocol for Anxiety in Children and Adolescents. REBT Treat. Anxiety Disord. Child. Adults. Springer, Cham; 2016 [cited 2017 Jul 17]. p. 51-67. Available from: https://link.springer.com/chapter/10.1007/978-3-319-18419-7_4

44. Iftene F, Predescu E, Stefan S, David D. Rational-emotive and cognitivebehavior therapy (REBT/CBT) versus pharmacotherapy versus REBT/CBT plus pharmacotherapy in the treatment of major depressive disorder in youth; a randomized clinical trial. Psychiatry Res. 2015;225:687-94.

45. March JS, Parker JD, Sullivan K, Stallings P, Conners CK. The multidimensional anxiety scale for children (MASC): factor structure, reliability, and validity. J Am Acad Child Adolesc Psychiatry. 1997;36:554-65.

46. Goodman R. The strengths and difficulties questionnaire: a research note. J Child Psychol Psychiatry. 1997;38:581-6.

47. Goodman A, Goodman R. Strengths and difficulties questionnaire as a dimensional measure of child mental health. J Am Acad Child Adolesc Psychiatry. 2009;48:400-3.

48. Hogendoorn SM, Wolters LH, Vervoort L, Prins PJM, Boer F, Kooij E, et al. Measuring negative and positive thoughts in children: an adaptation of the Children's automatic thoughts scale (CATS). Cogn Ther Res. 2010:34:467-78.

49. Ravens-Sieberer U, Bullinger M. News from the KINDL-questionnaire: a new version for adolescents. Qual Life Res. 1998:653-3.

50. Devilly GJ, Borkovec TD. Psychometric properties of the credibility/ expectancy questionnaire. J Behav Ther Exp Psychiatry. 2000;31:73-86.

51. Vigerland S, Ljótsson B, Thulin U, Öst L-G, Andersson G, Serlachius E. Internet-delivered cognitive behavioural therapy for children with anxiety disorders: a randomised controlled trial. Behav Res Ther. 2016;76:47-56.

52. Cohen J. Statistical power analysis for the behavioral sciences. Hilsdale NJ Lawrence Earlbaum Assoc. 1988:2.

53. Hayes AF. Introduction to mediation, Moderation, and Conditional Process Analysis: A Regression-Based Approach. Guilford Press; 2013.

54. General Assembly of the World Medical Association. World medical association declaration of Helsinki: ethical principles for medical research involving human subjects. J Am Coll Dent. 2014;81:14-8.

55. Murphy JM, Abel MR, Hoover S, Jellinek M, Fazel M. Scope, Scale, and Dose of the World's Largest School-Based Mental Health Programs. Harv. Rev. Psychiatry. 2017 [cited 2017 Sep 7];Publish Ahead of Print. Available from: http://journals.lww.com/hrpjournal/Abstract/publishahead/Scope,_Scale, and_Dose_of_the_World_s_Largest.99965.aspx.

\section{Ready to submit your research? Choose BMC and benefit from:}

- fast, convenient online submission

- thorough peer review by experienced researchers in your field

- rapid publication on acceptance

- support for research data, including large and complex data types

- gold Open Access which fosters wider collaboration and increased citations

- maximum visibility for your research: over $100 \mathrm{M}$ website views per year

At BMC, research is always in progress.

Learn more biomedcentral.com/submissions 